\title{
THE RESEARCH OF SOLUTION OF LEVINSON - SMITH EQUATION
}

O.G. Styrt

A.P. Krishchenko oleg_styrt@mail.ru

apkri@bmstu.ru

\section{Bauman Moscow State Technical University, Moscow, Russian Federation}

\begin{abstract}
We research the behavior of solutions of Levinson - Smith equation. In the case of an unperturbed system, friction is supposed to be positive. We consider the behavior of trajectories with respect to one localizing set that is, subset containing all compact invariant sets. More exactly, we show that this set is positively invariant and obtain some sufficient conditions for any trajectory to enter it. In the case of a perturbed system, we suggest that friction is lower bounded by some positive number and perturbation is a bounded continuous function. Similarly, we consider one localizing set in terms of non-autonomous systems and prove that it is positively invariant
\end{abstract}

\section{Keywords}

Dynamical system, localization, compact invariant set

Received 20.06.2016

(C) Bauman Moscow State Technical University, 2017

The authors were supported by the Ministry of education and science of the Russian Federation (project 1.644.2014/K and project 736 of the program "Organization of research")

Introduction. In this work, we consider Levinson - Smith equations of order two [1, 2]

$$
\ddot{x}+f(x, \dot{x}) \dot{x}+g(x)=e(t)
$$

researched in different assumptions on the functions $f(x, \dot{x}), g(x)$, and $e(t)$ in a number of papers. First of all, in the monograph [3] devoted to qualitative geometric analysis of differential equations, especial attention is payed on equations of order two. Among the rest, the equation (1) and its particular case without perturbation $e(t)$ are studied in detail. Another particular case is researched with many aspects the Li'enard equation

$$
\ddot{x}+f(x) \dot{x}+g(x)=0 .
$$

In [4], the qualitative behavior of trajectories of this equation is studied from the viewpoint of boundedness, oscillation, and periodicity. In $[5,6]$, upper bounds for the amplitude of limit cycles of the equation (2) are obtained. In [7], the period function of the equation (2) is researched in the suggestion that the origin $O$ is its equilibrium point of the "center" type. In this assumption, this function is defined in $D \backslash\{O\}$, where $D$ is the largest domain containing $O$ and consisting completely of cycles surrounding $O$. Besides, the paper [8] is concerned with a generalization of the Li'enard equation the perturbed equation

$$
\ddot{x}+f(x) \dot{x}+g(x)=e(t) .
$$


We will further suppose that $f$ and $g$ in (1) are continuous and Lipschitz functions, the function $e$ is continuous and bounded, and, unlike the most of famous works, we will also suggest that the function

$$
G(x) \stackrel{\operatorname{def}}{=} \int_{0}^{x} g(x) d x
$$

is upper bounded. Thus, $G^{*} \stackrel{\text { def }}{=} \sup \{G(x)\}_{x \in \mathbb{R}}<+\infty$.

In these and some other assumptions, the paper makes qualitative research of behavior of solutions of the system (1) based on the method of localizing compact invariant sets of dynamical systems [9-13].

The general form of the localization problem. Let us briefly describe this localizing method applied in analysis of different nonlinear systems [14-16].

Consider a dynamical system

$$
\dot{z}=q(z)
$$

where $z \in \mathbb{R}^{n}, q(z)=\left(q_{1}(z), \ldots, q_{n}(z)\right)^{\mathrm{T}}$, and $q(z)$ is a Lipschitz function.

A subset $M \subset \mathbb{R}^{n}$ is called an invariant set of the system (3) if, for each point $z_{0} \in M$, the trajectory $z\left(t, z_{0}\right)$ of the system (3) passing through the point $z_{0}$ is contained in $M$. The localization problem consists in finding sets (localizing sets) in $\mathbb{R}^{n}$ containing all compact invariant sets of the system (3) [9-11].

Let $\varphi$ be an arbitrary function in $C^{1}\left(\mathbb{R}^{n}\right)$ and

$$
\dot{\varphi}(z)=\sum_{i=1}^{\operatorname{def}} q_{i}(z) \frac{\partial \varphi(z)}{\partial z_{i}}
$$

the derivative of $\varphi$ with respect to the system (3). The subset

$$
S_{\varphi} \stackrel{\text { def }}{=}\left\{z \in \mathbb{R}^{n}: \dot{\varphi}(z)=0\right\}
$$

is called the universal section. Set $\varphi_{\text {sup }} \stackrel{\text { def }}{=} \sup _{z \in S_{\varphi}}\{\varphi(z)\}, \quad \varphi_{\text {inf }} \stackrel{\text { def }}{=} \inf _{z \in S_{\varphi}}\{\varphi(z)\}$. Then all compact invariant sets of the system (3) are contained in the subset [9-11]

$$
\Omega(\varphi) \stackrel{\text { def }}{=}\left\{z \in \mathbb{R}^{n}: \varphi_{\text {inf }} \leq \varphi(z) \leq \varphi_{\text {sup }}\right\} .
$$

In other words, this subset is localizing for the system (3). The above-mentioned function $\varphi$ is called localizing.

This method of localizing compact invariant sets is also applicable in a more general case of non-autonomous systems

$$
\dot{z}=q(z, t)
$$

where $z \in \mathbb{R}^{n}, \quad t \in \mathbb{R}, q(z, t)=\left(q_{1}(z, t), \ldots, q_{n}(z, t)\right)^{\mathrm{T}}$, and $q(z, t)$ is a continuous and $z$-Lipschitz function. 
A subset $M \subset \mathbb{R}^{n}$ is called an invariant set of the system (4) if, for each point $z_{0} \in M$, there exists a number $t_{0} \in \mathbb{R}$ such that the trajectory of the system (4) passing through the point $z_{0}$ at the time $t_{0}$ is contained in $M$. In the non-autonomous case, the localization problem also consists in finding localizing sets (subsets in $\mathbb{R}^{n}$ containing all compact invariant sets of the system $(4)[12,13])$.

In the non-autonomous case, we also take a localizing function $\varphi \in C^{1}\left(\mathbb{R}^{n}\right)$. Its derivative with respect to the system (4) is

$$
\frac{d \varphi}{d t}(z, t)=\sum_{i=1}^{n} q_{i}(z, t) \frac{\partial \varphi(z)}{\partial z_{i}} .
$$

The universal section is the subset

$$
s_{\varphi} \stackrel{\operatorname{def}}{=}\left\{z \in \mathbb{R}^{n}: \exists t \in \mathbb{R} \frac{d \varphi}{d t}(z, t)=0\right\} .
$$

Like in the autonomous case, the subset

$$
\Omega(\varphi) \stackrel{\text { def }}{=}\left\{z \in \mathbb{R}^{n}: \varphi_{\text {inf }} \leq \varphi(z) \leq \varphi_{\text {sup }}\right\},
$$

where $\varphi_{\text {sup }} \stackrel{\text { def }}{=} \sup _{z \in s_{\varphi}}\{\varphi(z)\}, \quad \varphi_{\text {inf }} \stackrel{\text { def }}{=} \inf _{z \in s_{\varphi}}\{\varphi(z)\}$, is localizing for the system $(4)[12,13]$.

The case of positive friction. Let us pass to constructing a localizing set of the unperturbed Levinson - Smith system

$$
\begin{gathered}
\dot{x}=y \\
\dot{y}=-f(x, y) y-g(x)
\end{gathered}
$$

supposing $f>0$ in $\mathbb{R}^{2}$.

Take a localizing function

$$
h=h(x, y) \stackrel{\text { def }}{=} \frac{y^{2}}{2}+G(x) .
$$

Its derivative with respect to the system (5) is

$$
\dot{h}(x, y)=y \dot{y}+G^{\prime}(x) \dot{x}=y(-f(x, y) y-g(x))+g(x) y=-f(x, y) y^{2} .
$$

The function $\dot{h}(x, y)=-f(x, y) y^{2}$ is non-positive, and the set of its zeros (i. e. the universal section $S_{h}$ ) is $O x$. Hence,

$$
\begin{aligned}
& h_{\mathrm{inf}} \stackrel{\text { def }}{=} \inf _{(x, y) \in S_{h}}\{h(x, y)\}=\inf _{x \in \mathbb{R}}\{h(x, 0)\}=\inf _{x \in \mathbb{R}}\{G(x)\} ; \\
& h_{\text {sup }} \stackrel{\operatorname{def}}{=} \sup _{(x, y) \in S_{h}}\{h(x, y)\}=\sup _{x \in \mathbb{R}}\{h(x, 0)\}=\sup _{x \in \mathbb{R}}\{G(x)\}=G^{*} .
\end{aligned}
$$


The inequality

$$
\inf \{G(x)\}_{x \in \mathbb{R}} \leq \frac{y^{2}}{2}+G(x)
$$

is always true, and, thus, the localizing set is

$$
\Omega \stackrel{\operatorname{def}}{=}\left\{(x, y) \in \mathbb{R}^{2}: \frac{y^{2}}{2}+G(x) \leq G^{*}\right\} .
$$

We have $G(x) \leq G^{*}$; so, $\Omega=\left\{(x, y) \in \mathbb{R}^{2}:|y| \leq \omega(x)\right\}, \omega(x) \stackrel{\operatorname{def}}{=} \sqrt{2\left(G^{*}-G(x)\right)}$. The graphs $\Gamma_{ \pm}$of the functions $y= \pm \omega(x)$ are located in the upper and the lower semiplains respectively symmetrically to each other across the axis $O x$, and the bound of the localizing set equals $\partial \Omega=\Gamma_{+} \cup \Gamma_{-}$. Also,

$$
\stackrel{\text { def }}{=} \Gamma_{+} \cap \Gamma_{-}=\left\{(x, 0) \in \mathbb{R}^{2}: x \in K\right\},
$$

where the set $K \stackrel{\text { def }}{=}\left\{x \in \mathbb{R}: G(x)=G^{*}\right\}$ consists of all global maximum points of the function $G(x)$.

The set $U \stackrel{\text { def }}{=} \mathbb{R}^{2} \backslash \Omega$ is defined by the inequality $|y|>\omega(x)$ and has two connected components $U_{+}$and $U_{-}$given by the inequalities $y>\omega(x)$ and $y<-\omega(x)$ respectively. The set $\bar{U} \subset \mathbb{R}^{2}$ is defined by the inequality $|y| \geq \omega(x)$, and, thus, $\bar{U} \cap\{O x\}=\Gamma$.

The set $\mathbb{R} \backslash K=\left\{x \in \mathbb{R}: G(x)<G^{*}\right\}$, being open, is the union of some countable family $\mathcal{P}$ of pairwise disjoint intervals. The connected components of the set $\Omega \backslash \Gamma$ are exactly all the subsets of the form

$$
\Omega_{I} \stackrel{\text { def }}{=}\{(x, y) \in \Omega: x \in I\}=\left\{(x, y) \in \mathbb{R}^{2}: x \in I,|y| \leq \omega(x)\right\}, I \in \mathcal{P} .
$$

Statement 1. The function $h$ is decreasing on each trajectory of the system (5).

4 The proof follows from non-positivity of the function $\dot{h}$.

Corollary 1. The set $\Omega$ is positively invariant. In other words, any trajectory, once entering $\Omega$, never leaves it.

४The set $\Omega$ is given by the inequality $h \leq G^{*}$. It remains to apply Statement 1 .

Corollary 2. The bound $\partial \Omega$ of the set $\Omega$ is semipermeable: trajectories can intersect it only entering the localizing set $\Omega$.

The set of all equilibrium points is the set $\Gamma_{0} \stackrel{\text { def }}{=}\left\{(x, 0) \in \mathbb{R}^{2}: x \in K_{0}\right\}$,

$$
K_{0} \stackrel{\text { def }}{=}\{x \in \mathbb{R}: g(x)=0\} \subset \mathbb{R} .
$$

If $x \in K$, then $x$ is a global maximum point of the function $G$, implying $g(x)=G^{\prime}(x)=0$ and $x \in K_{0}$. Thus, $K \subset K_{0}$. It follows that $\Gamma \subset \Gamma_{0}$. According to 
Corollary 1 , the set $\Omega \backslash \Gamma$ is positively invariant, and, therefore, so are all of its connected components $\Omega_{I}, I \in \mathcal{P}$.

From above, we conclude the following.

Statement 2. All the sets $\Omega_{I}, I \in \mathcal{P}$, are positively invariant.

For an arbitrary $C>0$, denote by $U_{+}(C)$ the subset defined by the inequalities $x>0$ and $\omega(x)<y<\omega(x)+C$ and by $U_{-}(C)$ the subset defined by the inequalities $x<0$ and $-\omega(x)-C<y<-\omega(x)$.

The following theorem is the main result of the paper.

Theorem 1. Suppose that the system (5) satisfies

$$
\forall C>0 \quad \inf \left\{f(p): p \in U_{+}(C)\right\}>0, \quad \inf \left\{f(p): p \in U_{-}(C)\right\}>0 .
$$

If $\gamma: t \rightarrow(x(t), y(t)), t \geq 0$, is an arbitrary trajectory, then $\gamma$ enters the localizing set $\Omega$ at some time $t_{0} \geq 0$ and then never leaves it, or $\gamma$ is completely located outside the localizing set $\Omega, h(\gamma(t)) \underset{t \rightarrow+\infty}{\longrightarrow} G^{*}, \operatorname{dist}(\gamma(t), \Omega) \underset{t \rightarrow+\infty}{\longrightarrow} 0 \quad(\operatorname{dist}(P, M)$ is the distance from the point $P$ to the set $M)$ and one of the following three conditions holds

1) $\gamma(t) \underset{t \rightarrow+\infty}{\longrightarrow} p \in \Gamma$;

2) $x(t) \underset{t \rightarrow+\infty}{\longrightarrow}+\infty$ and the integral $\int_{0}^{+\infty} \sqrt{G^{*}-G(x)} d x$ is convergent;

3) $x(t) \underset{t \rightarrow+\infty}{\longrightarrow}-\infty$ and the integral $\int_{-\infty}^{0} \sqrt{G^{*}-G(x)} d x$ is convergent.

4 If the trajectory enters the localizing set $\Omega$ at some time $t_{0} \geq 0$, then it never leaves it since the set $\Omega$ is positively invariant.

Suppose that $\gamma$ is completely located outside the localizing set $\Omega$. This means that $\gamma$ is contained in the set $U$ and, therefore, in one of its connected components $U_{+}$and $U_{-}$. Then, there are two possible cases

1) $\gamma \subset U_{+}$and, thus, $\dot{x}=y>\omega(x) \geq 0$, on $\gamma$, i. e. $x(t)$ is strictly increasing and, hence, $x(t) \underset{t \rightarrow+\infty}{\longrightarrow} x^{*} \in(-\infty ;+\infty]$;

2) $\gamma \subset U_{-}$and, thus, $\dot{x}=y<-\omega(x) \leq 0$ on $\gamma$, i. e. $x(t)$ is strictly decreasing and, hence, $x(t) \underset{t \rightarrow+\infty}{\longrightarrow} x^{*} \in[-\infty ;+\infty)$.

In both cases, the function $x(t)$ is strictly monotonous, $x(t) \underset{t \rightarrow+\infty}{\longrightarrow} x^{*} \in[-\infty ;+\infty]$, and the function $y(t)$ is nonzero and of constant sign on $\mathbb{R}$.

The set $\Omega$ is defined by each of two equivalent inequalities $|y| \leq \omega(x)$ and $h \leq G^{*}$. Hence, $|y|>\omega(x)$ and $h>G^{*}$ on $\gamma$. By Statement 1 , the function $h$ is decreasing on $\gamma$. Thus, $h(\gamma(t)) \longrightarrow{ }_{t \rightarrow+\infty}^{\longrightarrow} h^{*} \in\left[G^{*} ;+\infty\right)$ and $h \geq h^{*}$ on $\gamma$.

Lemma 1. If $\gamma$ is completely located outside the localizing set $\Omega$ and $x^{*}$ is a finite number, then the trajectory $\gamma$ tends to some point $p \in \Gamma$ and, also, $\operatorname{dist}(\gamma(t), \Omega) \underset{t \rightarrow+\infty}{\longrightarrow} 0, h(\gamma(t)) \underset{t \rightarrow+\infty}{\longrightarrow} h^{*}=G^{*}$. 
We have $(y(t))^{2}=2(h(\gamma(t))-G(x(t))) \underset{t \rightarrow+\infty}{\longrightarrow} d=2\left(h^{*}-G\left(x^{*}\right)\right)$. Since the function $y(t)$ is of constant sign, $y(t) \underset{t \rightarrow+\infty}{\longrightarrow} y^{*} \in(-\infty ;+\infty)$. Consequently, the trajectory $\gamma$ tends to the point $p=\left(x^{*}, y^{*}\right) \in \mathbb{R}^{2}$. Note that $p \in \Gamma_{0} \subset\{O x\}$. Besides, $\gamma \subset U$ implying $p \in \bar{U}, \quad p \in \bar{U} \cap\{O x\}=\Gamma$. Thus, $\gamma(t) \underset{t \rightarrow+\infty}{\longrightarrow} p \in \Gamma=\partial \Omega \cap\{O x\}$ and, therefore, $h(p)=G^{*}$. Also, $h^{*}=\lim _{t \rightarrow+\infty} h(\gamma(t))=h(p)=G^{*}$.

We see that, if $\gamma$ is completely located outside the localizing set $\Omega$ and $x^{*}$ is a finite number, then the first case of Theorem 1 takes place.

Now suppose that $\left|x^{*}\right|=\infty$.

Statement 3. If $(x, y) \in \mathbb{R}^{2}$ and $C \stackrel{\text { def }}{=} h(x, y)-G^{*} \geq 0$, then $|y| \leq \sqrt{2 C}+\omega(x)$.

We have $y^{2}=2(h(x, y)-G(x))=2 C+(\omega(x))^{2} \leq(\sqrt{2 C}+\omega(x))^{2}, \quad|y| \leq \sqrt{2 C}+$ $+\omega(x)$.

Proposition 1. If $\gamma$ is completely located outside the localizing set $\Omega$, then $\omega(x)<|y| \leq \sqrt{2\left(h-G^{*}\right)}+\omega(x)$ on $\gamma$.

On $\gamma$, we have $|y|>\omega(x)$ and, also, $h>G^{*}, h-G^{*}>0$, and, by Statement 3, $|y| \leq \sqrt{2\left(h-G^{*}\right)}+\omega(x)$

Corollary 3. If $\gamma$ is completely located outside the localizing set $\Omega$, then there exists a number $C>0$ such that $|y|<\omega(x)+C$ on $\gamma$ once $t \geq 0$.

The proof follows from Proposition 1 and decreasing of the function $h$ on $\gamma$.

Lemma 2. If $\gamma$ is completely located outside the localizing set $\Omega$ and $x^{\star}=+\infty$ $\left(x^{*}=-\infty\right)$, then there exist numbers $t_{0} \geq 0$ and $C>0$ such that, for each $t \geq t_{0}$, we have $\gamma(t) \in U_{+}(C) \quad\left(\gamma(t) \in U_{-}(C)\right)$.

By Corollary 3, there exists a number $C>0$ such that $-\omega(x)-C<y<\omega(x)+C$ on $\gamma$ once $t \geq 0$.

Suppose that $x^{*}=+\infty$. Then $\gamma \subset U_{+}$and, thus, $y>\omega(x)$ on $\gamma$. Besides, there exists a number $t_{0} \geq 0$ such that $x>0$ on $\gamma$ once $t \geq t_{0}$. If $(x, y)=\gamma(t), t \geq t_{0}$, then $x>0$ and $\omega(x)<y<\omega(x)+C$ implying $(x, y) \in U_{+}(C)$.

Now assume that $x^{*}=-\infty$. Then $\gamma \subset U_{-}$and, thus, $y<-\omega(x)$ on $\gamma$. Besides, there exists a number $t_{0} \geq 0$ such that $x<0$ on $\gamma$ once $t \geq t_{0}$. If $(x, y)=\gamma(t), t \geq t_{0}$, then $x<0$ and $-\omega(x)-C<y<-\omega(x)$ implying $(x, y) \in U_{-}(C)$.

Lemma 3. If the condition (6) holds, the trajectory $\gamma$ is completely located outside the localizing set $\Omega$, and $\left|x^{*}\right|=\infty$, then $h^{*}=G^{*}$, and the integral $\int_{0}^{x} \sqrt{G^{*}-G(x)} d x$ is convergent. 
Without loss of generality, we can assume that $x^{*}=+\infty$.

It follows from Lemma 2 and the condition (6) that, for $\gamma$, there exist numbers $t_{0} \geq 0$ and $\delta>0$ such that $f \geq \delta$ on $\gamma$ once $t \geq t_{0}$. Since $\int_{t_{0}}^{+\infty} f y^{2} d t=\int_{t_{0}}^{+\infty}(-\dot{h}) d t=$ $=\left.h\right|_{+\infty} ^{t_{0}}=h\left(t_{0}\right)-h^{*}$, the integral $\int_{t_{0}}^{+\infty} f y^{2} d t$ is convergent. On the other hand, $f \geq \delta$, $f y^{2} \geq \delta y^{2} \geq 0$ once $t \geq t_{0}$ and, thus, the integral $\int_{t_{0}}^{+\infty} \delta y^{2} d t$ is convergent. Hence, the integrals $\int_{t_{0}}^{+\infty} y^{2} d t$ and $\int_{t_{0}}^{+\infty}(h-G(x)) d t=\int_{t_{0}}^{+\infty} \frac{y^{2}}{2} d t$ are also convergent.

On $\gamma$, we have $h \geq h^{*} \geq G^{*} \geq G(x)$ implying $h-G(x) \geq h^{*}-G^{*} \geq 0$. Since the integral $\int_{t_{0}}^{+\infty}(h-G(x)) d t$ is convergent, $h^{*}=G^{*}$. Therefore, by Proposition 1, $\operatorname{dist}(\gamma(t), \Omega) \underset{t \rightarrow+\infty}{\longrightarrow} 0$.

As said above, on $\gamma$, we have $h \geq G^{*} \geq G(x), \quad h-G(x) \geq G^{*}-G(x) \geq 0$, $\sqrt{h-G(x)} \geq \sqrt{G^{*}-G(x)} \geq 0, \quad \frac{y^{2}}{\sqrt{2}}=\sqrt{h-G(x)}|y| \geq \sqrt{G^{*}-G(x)}|y| \geq 0$, and, since the integral $\int_{t_{0}}^{+\infty} y^{2} d t$ is convergent, so is the integral $\int_{t_{0}}^{+\infty} \sqrt{G^{*}-G(x)}|y| d t$. Also, recall that the function $y(t)=\dot{x}(t)$ is of constant sign and the function $x(t)$ is strictly monotonous. It follows from above that the integral $\int_{t_{0}}^{+\infty} \sqrt{G^{*}-G(x)} y d t=$ $=\int_{x\left(t_{0}\right)}^{x^{*}} \sqrt{G^{*}-G(x)} d x$ is convergent and, thus, so is the integral $\int_{0}^{x^{*}} \sqrt{G^{*}-G(x)} d x$.

This completely proves Theorem 1 .

Corollaries and examples. Corollary 4. Suppose that the condition (6) holds and the integrals $\int_{0}^{+\infty} \sqrt{G^{*}-G(x)} d x$ and $\int_{-\infty}^{0} \sqrt{G^{*}-G(x)} d x$ are divergent. Then any trajectory either enters the set $\Omega$ and then never leaves it or tends to some equilibrium point $p \in \Gamma$.

Corollary 5. Suppose that the condition (6) holds, the function $g$ has period $T>0$, $g \not 0$, and $\int_{0}^{T} g(t) d t=0$. Then any trajectory either enters the set $\Omega$ and then never leaves it or tends to some equilibrium point $p \in \Gamma$.

4 The function $G(x)$ is upper bounded since, for all $x \in \mathbb{R}$, 


$$
|G(x)|=\left|\int_{0}^{x} g(t) d t\right| \leq T \max \{|g(x)|\} .
$$

Thus, $G^{*}=\sup \{G(x)\}_{x \in \mathbb{R}}<+\infty$. The function $G$ is periodic and $G \not \exists G^{*}$. Consequently, the integrals $\int_{0}^{+\infty} \sqrt{G^{*}-G(x)} d x$ and $\int_{-\infty}^{0} \sqrt{G^{*}-G(x)} d x$ are divergent. It remains to apply Corollary 4 .

Now we give one more corollary of Proposition 1.

Corollary 6. If $\gamma$ is completely located outside the localizing set $\Omega$ and $h^{*}=G^{*}$, then $|y|-\omega(x) \underset{t \rightarrow+\infty}{\longrightarrow} 0$, i.e. $\operatorname{dist}(\gamma(t), \Omega) \underset{t \rightarrow+\infty}{\longrightarrow} 0$.

Example 1. Consider the equation $\ddot{x}+\frac{1}{\pi+x+\dot{x}^{2}} \dot{x}+\sin x=0$. In the notations above, we have $f(x, y)=\frac{1}{\pi+x+y^{2}}, \quad g(x)=\sin x$. Hence, $\quad G(x)=1-\cos x$, $G^{*}=2<+\infty$. The localizing set $\Omega \subset \mathbb{R}^{2}$ is defined by the inequality $y^{2} / 2-\cos x \leq 1$. Besides, $\Gamma=\partial \Omega \cap(O x)=\left\{(x, 0) \in \mathbb{R}^{2}: \cos x=-1\right\}=\left\{((2 n+1) \pi, 0) \in \mathbb{R}^{2}: n \in \mathbb{Z}\right\}$.

For each $x \in \mathbb{R}$, we have $G(x) \geq 0, G^{*}-G(x) \leq 2, \omega(x)=\sqrt{2\left(G^{*}-G(x)\right)} \leq 2$. Take an arbitrary number $C>0$. If $(x, y) \in U_{ \pm}(C)$, then $|y|<\omega(x)+C \leq 2+C, \pi+x+$ def $+y^{2}<M=3 \pi / 2+(2+C)^{2}, f(x, y)>1 / M$. Thus, $\inf \left\{f(p): p \in U_{ \pm}(C)\right\} \geq 1 / M>0$. Since the number $C>0$ is chosen arbitrarily, the condition (6) holds and, by Corollary 5, any trajectory either tends to some equilibrium point $P_{n}((2 n+1) \pi, 0) \in \Gamma$, $n \in \mathbb{Z}$, of the "saddle" type or enters the localizing set $\Omega$ and then never leaves it.

The equilibrium points $P_{n}$ divide the set $\Omega$ into the bounded positively invariant sets

$$
\Omega_{n} \stackrel{\text { def }}{=}\{(x, y) \in \Omega:|x-2 \pi n|<\pi\}, \quad n \in \mathbb{Z} .
$$

Each of the sets $\Omega_{n}$ contains one stable equilibrium point $(2 \pi n, 0)$. By LaSalle theorem, this equilibrium point attracts all trajectories in $\Omega_{n}$.

It follows from above that any trajectory either tends to one of the equilibrium points $((2 n+1) \pi, 0)$ or enters a set $\Omega_{n}$ and then, not leaving it, tends to the stable equilibrium point $(2 \pi n, 0)$.

Remark. If the condition (6) holds, the function $g$ has period $T$, and $\int_{0}^{T} g(t) d t=0$, then the system (5) does not have periodic trajectories and each of its trajectories tends to one of its equilibrium points.

Example 2. Consider the equation

$$
\ddot{x}+\dot{x}+\frac{x}{\left(1+x^{2}\right)^{2}}=0
$$

and prove its global asymptotic stability. 
The origin is an asymptotically stable equilibrium point if this system. Show that it attracts all trajectories of the system.

In the notations above, $f(x, y)=1, g(x)=\frac{x}{\left(1+x^{2}\right)^{2}}$. Thus, $G(x)=\frac{x^{2}}{2\left(1+x^{2}\right)}$, $G^{*}=1 / 2<+\infty$. Take a localizing function $h=\frac{y^{2}}{2}+G(x)$. The localizing set $\Omega \subset \mathbb{R}^{2}$ is defined by the inequality $h(x, y) \leq 1 / 2$, i. e.

$$
y^{2}+\frac{x^{2}}{1+x^{2}} \leq 1
$$

The condition (6) holds and, by Corollary 4, any trajectory enters localizing set $\Omega$ and then never leaves it.

Inside $\Omega$, we have $h(x, y)<1 / 2$. All sets $\left\{(x, y) \in \mathbb{R}^{2}: h(x, y) \leq c\right\}, c<1 / 2$ are bounded. They are also positively invariant since $\dot{h}(x, y)=-y^{2} \leq 0$.

By LaSalle theorem, the equilibrium point $(0,0)$ attracts all trajectories in $\Omega$.

A perturbed system. Consider the perturbed Levinson - Smith equation

$$
\begin{gathered}
\dot{x}=y ; \\
\dot{y}=-f(x, y) y-g(x)+e(t) .
\end{gathered}
$$

We suppose that $f$ and $g$ are Lipschitz functions. We also assume that $f$ is a function lower bounded by a number $\delta>0$ and $e$ is a bounded continuous function.

Set $G(x) \stackrel{\operatorname{def} x}{=} \int_{0}^{x} g(t) d t$. We have $G^{\prime}=g$. Suppose that $G^{*}=\operatorname{sef}\{G(x)\}_{x \in \mathbb{R}}<+\infty$. Since the function $e$ is bounded, its set of values $E \subset \mathbb{R}$ is contained in the segment $[-c ; c]$ for some $c>0$.

Take a localizing function $h=\frac{y^{2}}{2}+G(x)$. Its derivative with respect to the system (7) is

$$
\dot{h}=G^{\prime}(x) \dot{x}+y \dot{y}=g(x) y+y(-f(x, y) y-g(x)+e(t))=y(e(t)-f(x, y) y) .
$$

The universal section $s_{h}$ is contained in the union of the axis $O x$ and the set

$$
\begin{gathered}
\left\{(x, y) \in \mathbb{R}^{2}: f(x, y) y \in E\right\} \subset\left\{(x, y) \in \mathbb{R}^{2}:|f(x, y)| \cdot|y| \leq c\right\} \subset \\
\subset\left\{(x, y) \in \mathbb{R}^{2}: \delta|y| \leq c\right\} .
\end{gathered}
$$

Therefore, for each $(x, y) \in s_{h}$, we have $|y| \leq c / \delta, h(x, y) \leq \frac{c^{2}}{2 \delta^{2}}+G^{*}$. Hence,

$$
h_{\text {sup }} \stackrel{\text { def }}{=} \sup \{h(x, y)\}_{(x, y) \in s_{h}} \leq \frac{c^{2}}{2 \delta^{2}}+G^{*},
$$

and, thus, the corresponding localizing set is contained in the subset $\hat{\Omega} \subset \mathbb{R}^{2}$ defined by the inequality $\frac{y^{2}}{2}+G(x) \leq \frac{c^{2}}{2 \delta^{2}}+G^{*}$. Consequently, $\hat{\Omega}$ is a localizing set. 
We will further denote by $G_{c}^{*}$ the number $\frac{c^{2}}{2 \delta^{2}}+G^{*}$.

The set $\hat{\Omega}$ is given by the inequality $h(x, y) \leq G_{c}^{*}$.

Lemma 4. The derivative of the function $h$ with respect to the system (7) is nonpositive on the set defined by the inequality $h(x, y) \geq G_{c}^{*}$ and upper bounded by $a$ negative number on the set given by the inequality $h(x, y) \geq h_{0}$ for each $h_{0}>G_{c}^{*}$.

४Take an arbitrary number $h_{0}>G_{c}^{*}$. Set $\stackrel{\text { def }}{=} h_{0}-G_{c}^{*}>0$.

If $(x, y) \in \mathbb{R}^{2}, t \in \mathbb{R}$, and $h(x, y) \geq h_{0}$, then

$$
\frac{y^{2}}{2}+G^{*} \geq \frac{y^{2}}{2}+G(x) \geq h_{0}=G_{c}^{*}+\varepsilon=\frac{c^{2}}{2 \delta^{2}}+G^{*}+\varepsilon,
$$

i. e. $\delta|y| \geq \sqrt{c^{2}+2 \delta^{2} \varepsilon}$,

$$
\begin{gathered}
\dot{h}(x, y, t)=y(e(t)-f(x, y) y)=y e(t)-f(x, y) y^{2} \leq|y e(t)|-\delta y^{2}= \\
=|y| \cdot|e(t)|-\delta y^{2} \leq c|y|-\delta y^{2}=-|y|(\delta|y|-c) \leq-|y|\left(\sqrt{c^{2}+2 \delta^{2} \varepsilon}-c\right)
\end{gathered}
$$

and, therefore, $-\dot{h}(x, y, t) \geq \frac{1}{\delta}(\delta|y|)\left(\sqrt{c^{2}+2 \delta^{2} \varepsilon}-c\right) \geq \frac{1}{\delta} \sqrt{c^{2}+2 \delta^{2} \varepsilon}\left(\sqrt{c^{2}+2 \delta^{2} \varepsilon}-c\right)$.

Since $\varepsilon>0$, then the number $\frac{1}{\delta} \sqrt{c^{2}+2 \delta^{2} \varepsilon}\left(\sqrt{c^{2}+2 \delta^{2} \varepsilon}-c\right)$ is positive.

Corollary 7. The set $\hat{\Omega}$ is positively invariant.

४The bound $\partial \hat{\Omega}$ of the set $\hat{\Omega}$ is defined by the equation $h(x, y)=G_{c}^{*}$. By Lemma $4, \dot{h} \leq 0$ on $\partial \hat{\Omega}$. Hence, the bound $\partial \hat{\Omega}$ is semipermeable and trajectories can intersect it only in the direction of the set $\hat{\Omega}$.

Thus, any trajectory, once entering $\hat{\Omega}$, never leaves it. Also, on any trajectory never entering $\hat{\Omega}, h>G_{c}^{*}$ and, by Lemma 4 , the function $h$ is decreasing and tends to $G_{c}^{*}$.

Conclusion. In the paper, Levinson - Smith dynamical system was researched with the object to localizing compact invariant sets and the behavior of solutions with respect to one localizing set. In the case of an unperturbed system, with the assumption of positive friction, it was proved that this set is positively invariant, and some sufficient conditions for any trajectory to enter it were obtained. In the case of a perturbed system, with the suggestion that friction is lower bounded by some positive number and perturbation is a bounded continuous function, it was proved that this localizing set is positively invariant.

\section{REFERENCES}

[1] Levinson N., Smith O.K. A general equation for relaxation oscillations. Duke Mathematical Journal, 1942, vol. 9 (2), pp. 382-403.

[2] Levinson N. On the existence of periodic solutions for second order differential equations with a forcing term. J. of Math. and Phys., 1943, vol. 22, pp. 41-48. 
[3] Lefschetz S. Differential equations: Geometric theory. N.Y. - London, Interscience Publ., 1957. $374 \mathrm{p}$.

[4] Villari G. On the qualitative behavior of solutions of Li'enard equation. J. Differential Equations, 1987, vol. 67 (2), pp. 269-277.

[5] Lijuna Y., Xianwu Z. An upper bound for the amplitude of limit cycles in Li'enard systems with symmetry. J. Differential Equations, 2015, vol. 258 (8), pp. 2701-2710.

[6] Turner N., McClintock P.V.E., Stefanovska A. Maximum amplitude of limit cycles in Li'enard systems. Phys. Rev. E, 2015, vol. 91, p. 012927.

[7] Sabatini M. On the period function of Li'enard systems. J. Differential Equations, 1999, vol. 152 (2), pp. 467-487.

[8] Burton T.A., Townsend C.G. On the generalized Li'enard equation with forcing function. J. Differential Equations, 1968, vol. 4 (4), pp. 620-633.

[9] Krishchenko A.P. Localization of limit cycles. Differential Equations, 1995, vol. 31 (11), pp. 1826-1833.

[10] Krishchenko A.P. Estimations of domains with cycles. Comput. Math. Appl., 1997, vol. 34 (2-4), pp. 325-332.

[11] Krishchenko A.P. Localization of invariant compact sets of dynamical systems. Differential Equations, 2005, vol. 41 (12), pp. 1669-1676. DOI: 10.1007/s10625-006-0003-6

[12] Krishchenko A.P., Starkov K.E. Localization of compact invariant sets of nonlinear timevarying systems. Int. J. Bifur. Chaos Appl. Sci. Engrg., 2008, vol. 24 (5), pp. 1599-1604.

[13] Kanatnikov A.N., Krishchenko A.P. Localization of invariant compact sets of nonautonomous systems. Differential Equations, 2009, vol. 45 (1), pp. 46-52.

[14] Starkov K.E., Coria L.N. Global dynamics of the Kirschner - Panetta model for the tumor immunotherapy. Nonlin. Anal.: Real World Appl., 2013, vol. 14 (3), pp. 1425-1433.

[15] Starkov K.E., Villegas A. On some dynamical properties of a seven-dimensional cancer model with immunotherapy. Int. J. Bifur. Chaos, 2014, vol. 24 (02), p. 1450020.

[16] Krishchenko A.P., Starkov K.E. Dynamical analysis of Raychaudhuri equations based on the localization method of compact invariant sets. Int. J. Bifur. Chaos, 2014, vol. 24 (11), p. 1450136.

Styrt O.G. - Cand. Sci. (Phys.-Math.), Assoc. Professor of Mathematical Simulation Department, Bauman Moscow State Technical University (2-ya Baumanskaya ul. 5, Moscow, 105005 Russian Federation).

Krishchenko A.P. - Dr. Sci. (Phys.-Math.), Professor, Head of Mathematical Simulation Department, Bauman Moscow State Technical University (2-ya Baumanskaya ul. 5, Moscow, 105005 Russian Federation).

\section{Please cite this article in English as:}

Styrt O.G., Krishchenko A.P. The Research of Solution of Levinson - Smith Equation. Vestn. Mosk. Gos. Tekh. Univ. im. N.E. Baumana, Estestv. Nauki [Herald of the Bauman Moscow State Tech. Univ., Nat. Sci.], 2017, no. 1, pp. 15-25. DOI: 10.18698/1812-3368-2017-1-15-25 